\title{
CARACTERIZAÇÃO DE NÚCLEOS ESPONTÂNEOS DE Clidemia urceolata DC. EM ÁREAS PERTURBADAS DA MATA ATLÂNTICA
}

\section{CHARACTERIZATION OF SPONTANEOUS NUCLEI OF Clidemia urceolata DC. IN DISTURBED AREAS OF THE ATLANTIC FOREST}

\author{
Cristiana do Couto Miranda ${ }^{1}$ Ricardo Valcarcel ${ }^{2}$ Pablo Hugo Alves Figueiredo \\ Felipe Araújo Mateus ${ }^{4}$ Cristiane Roppa ${ }^{5}$ André Felippe Nunes-Freitas ${ }^{6}$
}

\begin{abstract}
RESUMO
A Clidemia urceolata DC. é uma espécie pioneira da família Melastomataceae, que coloniza espontaneamente pastagens abandonadas. Ela forma núcleos de tamanhos variados, onde podem ser observadas outras espécies de grupo sucessional mais avançado, podendo constituir uma espécie facilitadora. O presente estudo objetivou quantificar e caracterizar os núcleos na bacia hidrográfica do rio Barra Mansa

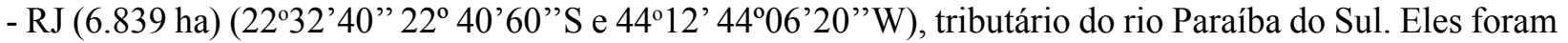
georreferenciados e caracterizados quanto ao porte dos indivíduos de Clidemia urceolata (baixo: $\mathrm{H} \leq 0,60$ $\mathrm{m}$; médio: $0,60<\mathrm{H}<1,20 \mathrm{~m}$; e alto: $\mathrm{H} \geq 1,20 \mathrm{~m}$ ), adensamento (esparso, médio e adensado), área do núcleo (pequeno: $\leq 500 \mathrm{~m}^{2}$; médio: 500 a $2000 \mathrm{~m}^{2}$; e grande: $\geq 2000 \mathrm{~m}^{2}$ ) e estágio de desenvolvimento (inicial, intermediário e avançado). Foram identificados 26 núcleos, totalizando 7,9 ha. Desses, houve predomínio de núcleos com indivíduos de porte médio (76,92\%), pouco adensado (42,31\%), tamanho grande $(42,31 \%)$ e estágio intermediário de desenvolvimento (46,16\%), evidenciando que se encontram em formação, e que a estratégia de colonização, reprodução e dispersão da Clidemia urceolata pode contribuir para dinamizar a sucessão ecológica, podendo atuar como agente espontâneo de restauração florestal das áreas perturbadas. Palavras-chave: pastagens abandonadas; espécie facilitadora; restauração florestal.
\end{abstract}

\begin{abstract}
Clidemia urceolata DC. is a pioneer species of the family Melastomataceae which colonizes spontaneously abandoned pastures. It forms nuclei of different sizes, where other species of advanced successional groups can be observed, constituting a facilitative species. The present study aimed to quantify and characterize the nuclei in the watershed of Barra Mansa river, Rio de Janeiro state (6.839 ha) (22 $32^{\prime} 40^{\prime \prime} 22^{\circ} 40^{\prime} 60^{\prime \prime} \mathrm{S}$ e $44^{\circ} 12^{\prime} 44^{\circ} 06^{\prime} 20^{\prime \prime} \mathrm{W}$ ), which is a tributary of Paraíba do Sul river. They were geographically referenced and characterized regarding to the size of Clidemia urceolata individuals (low: $\mathrm{H}<0,60 \mathrm{~m}$; medium: 0,60 $<\mathrm{H}<1,20 \mathrm{~m}$; and high: $\mathrm{H} \geq 1,20 \mathrm{~m}$ ), densification (scattered, medium and dense), area of the nuclei (small: $\leq 500 \mathrm{~m}^{2}$; medium: 500 to $2000 \mathrm{~m}^{2}$; and large: $\geq 2000 \mathrm{~m}^{2}$ ) and development stage (initial, intermediate and advanced). 26 nuclei were identified, adding up to 7,9 ha. There was the prevalence of nuclei with medium
\end{abstract}

1 Bióloga, Dra ${ }^{\mathrm{a}}$, Professora do Instituto Federal do Rio de Janeiro, Rua José Breves, 550, Centro, CEP 27197-000, Pinheiral (RJ), Brasil. cristiana.miranda@ifrj.edu.br

2 Engenheiro Florestal, Dr., Professor Associado IV do Departamento de Ciências Ambientais, Universidade Federal Rural do Rio de Janeiro, Rodovia BR 465, Km 7, CEP 23561-000, Seropédica (RJ), Brasil. ricardo.valcarcel@gmail.com

3 Estudante de Engenharia Florestal, Universidade Federal Rural do Rio de Janeiro, Rodovia BR 465, Km 7, CEP 23561-000, Seropédica (RJ), Brasil. pablo_figueiredo@yahoo.com.br

4 Engenheiro Florestal, Universidade Federal Rural do Rio de Janeiro, Rodovia BR 465, Km 7, CEP 23561-000, Seropédica (RJ), Brasil. felipearaujomateus@gmail.com

5 Engenheira Florestal, Doutoranda em Ciências Ambientais e Florestais, Universidade Federal Rural do Rio de Janeiro, Rodovia BR 465, Km 7, CEP 23561-000, Seropédica (RJ), Brasil. cristianeroppa@gmail.com

6 Biólogo, Dr., Professor Adjunto II do Departamento de Ciências Ambientais, Universidade Federal Rural do Rio de Janeiro, Rodovia BR 465, Km 7, CEP 23561-000, Seropédica (RJ), Brasil. afnfreitas@gmail.com

Recebido para publicação em 24/07/2012 e aceito em 14/06/2013

Ci. Fl., v. 25, n. 1, jan.-mar., 2015 
sized individuals $(76,92 \%)$, slightly dense $(42,31 \%)$, big area $(42,31 \%)$ and intermediate developmental stage $(46,16 \%)$, evidencing that they are in progress and that colonization strategy, reproduction and dispersion of Clidemia urceolata can contribute to boost the ecological succession, being able to act as a spontaneous agent of forest restoration of the disturbed areas.

Keywords: abandoned pastures; facilitative species; forest restoration.

\section{INTRODUÇÃO}

A supressão da vegetação associada aos usos inadequados dos solos tem contribuído para a formação de áreas perturbadas com tendência à degradação, ou seja, ecossistemas que sofrem empobrecimento paulatino no tempo, mesmo com mínimas taxas de uso. No entanto, apesar dessa condição, essas áreas ainda podem resguardar potencial biótico capaz de promover sua autorregeneração (CARVALHO, 2000; CARPANEZZI, 2005).

$\mathrm{Na}$ bacia hidrográfica do rio Paraíba do Sul, umas das principais bacias do Sistema hidrográfico brasileiro, as áreas perturbadas são oriundas do histórico de uso do solo, no qual os ciclos econômicos da cafeicultura, pecuária leiteira e de corte foram responsáveis pela supressão das florestas nativas e esgotamento dos solos, deixando-os empobrecidos. A inobservância de critérios conservacionistas promoveu perda do potencial produtivo dos solos, que atualmente têm capacidade de suporte para sustentar vegetação de porte herbáceo, rala e frágil. As extensas áreas em processo de degradação, com diferentes intensidades de processos erosivos, perda da qualidade da água e baixa produtividade agrícola, representam evidências da magnitude da perturbação e acarretam perdas de serviços ambientais deste ecossistema, como qualidade e disponibilidade hídricas (VALCARCEL, 1995), influenciando negativamente no desenvolvimento regional, o que, segundo a literatura, pode ser quantificado economicamente (COSTANZA et al., 1997).

As áreas perturbadas constituem a principal paisagem da região do terço médio da bacia hidrográfica do rio Paraíba do Sul, onde há pequenos fragmentos florestais isolados entre pastagens abandonadas (TOLEDO e PEREIRA, 2004). Nas regiões de relevo acidentado, os problemas de erosão das encostas são mais frequentes e intensos, como nas bacias do rio Barra Mansa e Ribeirão Cachimbal (CASTRO et al., 2002; CEIVAP, 2002).
Para reverter o atual quadro de degradação, faz-se necessário empregar modelos alternativos de recuperação nos ambientes perturbados, pois as comunidades encontram-se descapitalizadas e sem meios para investir em medidas de restauração florestal, cujo reflorestamento é a principal atividade. Assim, um projeto de restauração pode se iniciar pelo levantamento das espécies que conseguem subsistir e criar meios para estabelecer serviços ambientais aos ecossistemas. Segundo Chazdon (2008), níveis originais desses serviços podem ser restabelecidos com o processo de recuperação, em especial de forma natural.

De acordo com Valcarcel e Silva (2000), a restauração de áreas onde ainda há solos com traços de matéria orgânica, mosaicos de pequenos fragmentos florestais e vegetação rala com predomínio de pastagens abandonadas, requer estratégias especificas. Segundo esses autores, tais estratégias demandam o conhecimento das espécies invasoras espontâneas da região, que suportam as adversidades locais, como ataque de insetos, estresses hídrico e térmico, resistência às queimadas e pastoreio extensivo. A recuperação florestal pode ser limitada por fatores bióticos e abióticos. Dentre os fatores bióticos está a alta densidade de gramíneas, que inibe a germinação, crescimento e estabelecimento de espécies lenhosas (MIRITI, 1998; HOLL et al., 2000; CAMPOS, 2010; ORTEGA-PIECK et al., 2011); a exposição ao fogo (MARTINS et al., 2003); a destruição do banco de sementes viáveis (BAIDER et al., 1999; ARAUJO et al., 2001; GASPARINO et al., 2006; BRAGA et al., 2008); a redução da chuva de sementes (MIRITI, 1998; GASPARINO et al., 2006) e as altas taxas de predação destas por insetos e mamíferos (MOUTINHO, 1998). Já no contexto dos fatores abióticos, que restringem o estabelecimento de espécies florestais, estão incluídos a baixa umidade do solo e do ar, a compactação do solo, os valores elevados de temperatura e a baixa fertilidade, associada ao intenso escoamento superficial (BUSCHBACHER et al., 1988). A intensidade 
desses fatores é, em grande parte, determinada pela intensidade e modo de uso da terra antes do abandono (UHL et al., 1988; ENGEL e PARROTTA, 2008).

Algumas espécies exóticas têm sido utilizadas como iniciais no estabelecimento de processos sucessionais, principalmente pela sua rusticidade (CORTINES e VALCARCEL, 2009; ROPPA, 2009). No entanto, o grande desafio da restauração como ciência é ampliar o conjunto de espécies nativas rústicas o suficiente para colonizar, estabelecer populações viáveis e desenvolver propriedades emergentes, de modo a se tornarem espécies facilitadoras da sucessão ecológica, constituindo modelos naturais de restauração de ecossistemas perturbados.

$\mathrm{O}$ conhecimento das estratégias de estabelecimento e colonização adotadas pelas espécies vegetais pioneiras pode viabilizar projetos sustentáveis de restauração florestal. Nesse contexto, foi selecionada para este estudo, a espécie arbustiva Clidemia urceolata DC. (Melastomataceae), que se estabelece e coloniza espontaneamente pastagens abandonadas na bacia do rio Paraíba do Sul (MENEZES, 2008; MIRANDA et al., 2011). Nessas áreas, sua distribuição agregada forma núcleos de tamanhos variados, onde se observam espécies de diferentes hábitos, entre as quais se destaca Cecropia pachystachya Trécul (FIGUEIREDO et al., 2011). Dessa forma, a Clidemia urceolata pode exercer funções importantes no avanço da sucessão. Outras espécies pioneiras da mesma família ocorrem com frequência nos processos de regeneração natural dos ecossistemas florestais alterados (ARAÚJO et al., 2001; FRANCO, 2005; TRÊS et al., 2007; BRAGA et al., 2008), tendo importante função na reversão de tendência de degradação dos ecossistemas, pois são as primeiras a ativar mecanismos de resiliência. Por essas importantes habilidades, caracterizar os núcleos de Clidemia urceolata e sua distribuição ao longo das áreas perturbadas pode ser fundamental para aperfeiçoar estratégias de restauração ecológica.

O presente estudo objetivou quantificar e caracterizar os núcleos de vegetação espontânea com dominância fitofisionômica de Clidemia urceolata na microbacia do rio Barra Mansa de modo a avaliar seu potencial de colonização de pastagens abandonadas, em processo de degradação, de microbacias similares da bacia hidrográfica do rio Paraíba do Sul.

\section{MATERIAL E MÉTODOS}

\section{Área de Estudo}

A área estudada corresponde ao trecho médio e inferior da bacia hidrográfica do rio Barra Mansa (6.839 ha), pertencente ao Médio Paraíba do Sul. Ela se encontra no município de Barra Mansa -

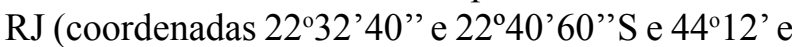
$44^{\circ} 06^{\prime} 20^{\prime \prime} \mathrm{W}$ ) (Figura 1). Segundo dados do INMET (1992), cuja série histórica remonta ao período 1962-1990, o clima é do tipo Aw, segundo Köppen, com médias anuais de precipitação de $1300 \mathrm{~mm}$ e de temperatura de $20,9^{\circ} \mathrm{C}$.

O histórico de devastação da Bacia hidrográfica do rio Barra Mansa reflete o processo de ocupação do Médio Paraíba, onde as florestas, que originalmente cobriam toda a região, foram substituídas pelas lavouras de café, no século XIX (DRUMMOND, 1997). A falta de critérios adequados de conservação do solo e de mananciais, caracterizada pelo desmatamento indiscriminado e plantios em linha no sentido do maior declive ao longo das encostas, contribuíram para decadência da cafeicultura no passado e, no presente, a formação de grandes extensões de terras degradadas com intensos processos erosivos, perda de biodiversidade, poluição, assoreamento dos cursos de água, redução na qualidade e disponibilidade de mananciais hídricos (COPPE-UFRJ, 2000). Em substituição às lavouras de café vieram as pastagens extensivas, que também não se caracterizaram pela adoção de técnicas de conservação dos solos e das águas (CEIVAP, 2002). Os desmatamentos das áreas declivosas aumentaram as fronteiras das pastagens (MENEZES, 2008) até a limitação de retorno econômico constituir o principal agente conservacionista regional, porém, com predomínio de pastagens abandonadas em processo de degradação.

A Bacia hidrográfica do rio Barra Mansa pertenceaobiomaMataAtlântica(RADAMBRASIL, 1983), cuja cobertura florestal encontra-se reduzida a aproximadamente 3\% (COPPE-UFRJ, 2000). Os remanescentes florestais têm composição estrutural semelhante à vegetação secundária (capoeiras). As pastagens são de baixa produtividade e altos níveis de degradação, ocupando mais de $80 \%$ da bacia, onde as cicatrizes dos processos erosivos constituem evidências práticas que atestam o estado de degradação (COPPE-UFRJ, 2000). Os solos predominantes nas encostas são Argissolo 


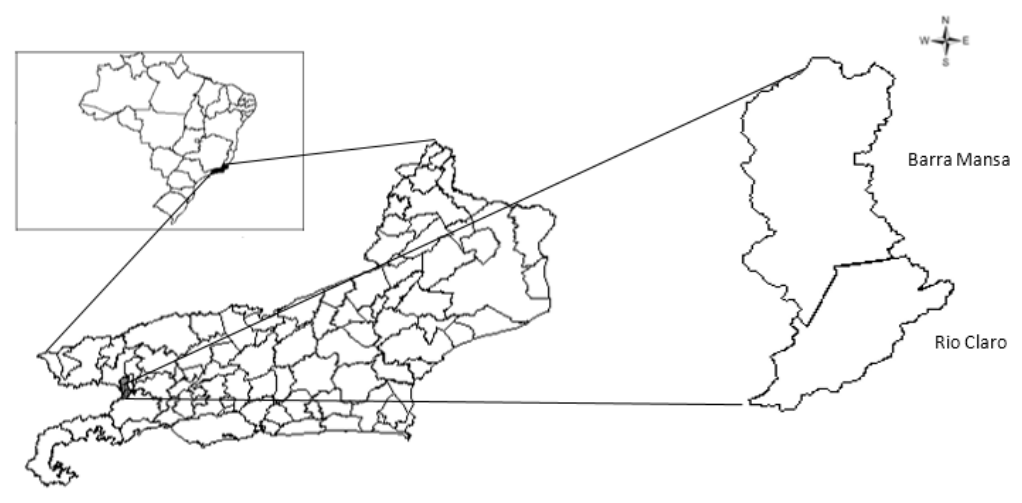

FIGURA 1: Localização da Bacia Hidrográfica do rio Barra Mansa - RJ, destacando o trecho do Município de Barra Mansa, onde foram feitos os levantamentos.

FIGURE 1: Location of the watershed of Barra Mansa River, RJ state, detaching the stretch of the municipal district of Barra Mansa, where the survey occurred.

Vermelho-Amarelo, Latossolo Amarelo e Argissolo Vermelho-Amarelo Planossólico (MENEZES, 1999). O relevo extremamente acidentado, associado à ocorrência de Argissolos de alta erodibilidade, são os ambientes em que se verificam os processos erosivos mais intensos (Fundação COPPETEC, 2007).

A prática da queimada, que está relacionada ao processo histórico de ocupação e "manejo das pastagens" da região, ainda é utilizada como modo barato de "renovação" das pastagens (COPPE-UFRJ, 2000). Estes processos se agravam pelas queimadas acidentais ou intencionais frequentes nas margens das rodovias. Essa prática resulta em contínuo empobrecimento dos solos e aumento dos processos erosivos, que, associado ao pisoteio do gado (sobrepastoreio) nas encostas declivosas, catalisam os processos erosivos, formando voçorocas (CASTRO et al., 2002).

A degradação do solo da bacia contribui para a reduzida importância econômica da atividade agropecuária em Barra Mansa, que é inferior a 3\% do PIB municipal (IBGE, 2011). Entre os anos de 1985 e 1996, esse município teve uma acentuada diminuição de áreas ocupadas com lavouras e pastagens (aproximadamente 23\%) (COPPE-UFRJ, 2000). A degradação do solo refletiu na evasão da população rural e no crescimento urbano, que se expandiu ocupando inadequadamente encostas com declives mais acentuados e margens de rios, que já se encontravam degradadas e sem condições geotécnicas que garantissem o controle da erosão

\section{(COPPE-UFRJ, 2000).}

\section{Espécie estudada}

A Clidemia urceolata DC. é um arbusto pioneiro, com porte variando entre de 0,5 a $2 \mathrm{~m}$ de altura, pertencente à família Melastomataceae (GOLDENBERG et al., 2005; MICHELANGELI e REGINATO, 2010) e conhecida regionalmente como mixirico. Essa família apresenta aproximadamente 4.570 espécies distribuídas pelas regiões tropicais e subtropicais (CLAUSING e RENNER, 2001). A maioria destas espécies ocorre nos Neotrópicos, sendo as formações florestais do leste do Brasil uma das regiões da América mais ricas em Melastomataceae (GOLDENBERG e REGINATO, 2006). Cerca de 175 destas espécies pertencem ao gênero Clidemia D. Don (RENNER, 1993; CANDIDO, 2005), que ocorre desde o sul do México até o sul do Brasil, interiorizando-se pelo continente até o Paraguai (WURDACK et al., 1993). Aproximadamente 50 espécies desse gênero ocorrem no Brasil (CANDIDO, 2005).

De acordo com Michelangeli e Reginato (2010), a Clidemia urceolata possui ampla distribuição da América Central ao Brasil, ocorrendo nos estados da Bahia, em toda a região sudeste, Paraná e Santa Catarina, dentro dos domínios fitogeográficos Caatinga e Mata Atlântica. Em pastagens abandonadas em processo de degradação da Bacia Hidrográfica do rio Paraíba do Sul é observada colonização espontânea da Clidemia 
urceolata (MENEZES, 2008; MIRANDA et al., 2011), formando núcleos de tamanhos variados.

De acordo com Goldenberg et al. (2005),

a Clidemia urceolata apresenta flores e frutos durante praticamente todo ano. Seus frutos carnosos contribuem para a dispersão zoocórica (LESSA e COSTA, 2009), que, segundo Levey (1990), é frequente nas espécies da família Melastomataceae. Ainda são poucos os estudos referentes à ecologia dessa espécie, especialmente quanto a sua função no processo de sucessão ecológica. Considerando seu potencial de colonização em áreas perturbadas, essa espécie foi selecionada como modelo de estudo de espécies que apresentam características funcionais semelhantes.

\section{Amostragem}

O levantamento dos núcleos de Clidemia urceolata (capoeira com dominância fitofisionômica de Clidemia urceolata DC) foi feito no trecho médio e inferior da bacia hidrográfica do rio Barra Mansa. Os núcleos foram caracterizados quanto ao porte médio dos indivíduos de Clidemia urceolata (porte baixo = plantas com $\mathrm{H} \leq 0,60 \mathrm{~m}$; médio $=0,60<\mathrm{H}<1,20 \mathrm{~m}$; e alto $=\mathrm{H} \geq 1,20 \mathrm{~m}$ ), adensamento (esparso $=$ possibilita acesso livre entre as plantas, dado o predomínio de pastagens; adensamento médio $=$ possibilita a entrada livre entre as plantas com maior grau de dificuldade, pois predomina a Clidemia urceolata; e adensado = não possibilita entrada livre), área do núcleo (pequeno: $\leq 500 \mathrm{~m}^{2}$; médio: entre 500 e $2000 \mathrm{~m}^{2}$ e grande: $\geq 2000 \mathrm{~m}^{2}$ ) e estágio de desenvolvimento do núcleo (inicial $=$ núcleo com predomínio de Clidemia urceolata e ausência de indivíduos arbóreos; intermediário $=$ predomínio da Clidemia urceolata e presença de indivíduos arbóreos $(\leq 10$ indivíduos arbóreos adultos)); e avançado = capoeira com indivíduos de Clidemia urceolata, mas com maior diversidade de espécies (> 10 indivíduos arbóreos).

As características dos núcleos de Clidemia urceolata, quanto ao porte das plantas, adensamento, área e estágio de desenvolvimento, foram avaliadas estatisticamente utilizando o teste Qui-quadrado (ZAR, 1999).

$\mathrm{A}$ área dos núcleos, georreferenciados em campo, com auxílio do GPS Garmim Etrex Venture, foi determinada a partir de imagens do Google Earth georreferenciadas no programa ArcMap (Arc GIS 9.2). Os pontos (núcleos georreferenciados) foram sobrepostos às imagens, segundo procedimento descrito por Figueiredo et al. (2002). A delimitação e a determinação da área dos núcleos de Clidemia urceolata foram realizadas por meio da ferramenta sketch tool do ArcMap.

\section{RESULTADOS E DISCUSSÃO}

Foram determinados 26 núcleos de vegetação com dominância fitofisionômica de Clidemia urceolata DC. em diferentes fases de desenvolvimento, que correspondem a 7,9 ha $(0,11 \%)$ do trecho médio e inferior da bacia hidrográfica estudada (Figura 2).

A maior parte dos núcleos $(76,96 \%)$ apresentou porte médio das plantas $\left(\chi^{2}=22,23, \mathrm{gl}\right.$ $=2, \mathrm{p}<0,01)$, ou seja, núcleos com predomínio de indivíduos de Clidemia urceolata com altura entre 0,6 e 1,2 m (Figura 3). Em relação ao adensamento, foi observada uma tendência de predomínio do médio (pouco adensado) $(42,31 \%)$ sobre o adensado $(34,61 \%)$, e por sua vez, sobre os esparsos $(23,08 \%)$, embora não tenha sido encontrada diferença significativa entre essas categorias $\left(\chi^{2}=1,46, \mathrm{gl}=\right.$ $2, p>0,05)$ (Figura 3). Esse mesmo comportamento foi observado para os parâmetros área e estágio de desenvolvimento. Apesar da ausência de diferenças significativas, foi observada uma tendência crescente de núcleos pequenos $(23,08 \%)$, médios $(34,61 \%)$ e grandes $(42,31 \%)\left(\chi^{2}=1,46, \mathrm{gl}=2\right.$, $\mathrm{p}>0,01)$ e de estágio de desenvolvimento avançado $(19,23 \%)$, inicial $(34,61 \%)$ e intermediário $(46,16 \%)$ $\left(\chi^{2}=2,85, \mathrm{gl}=2, \mathrm{p}>0,01\right)$ (Figura 3$)$.

Os resultados indicaram que os núcleos se encontram em processo de estabelecimento, predominando porte médio das plantas, pouco adensado, tamanho grande e estágio intermediário de desenvolvimento; com mais indivíduos de Clidemia urceolata e outras espécies entrando no sistema de pastagens abandonadas. O predomínio de núcleos com porte médio (indivíduos de Clidemia urceolata entre 0,6 e 1,2 $\mathrm{m}$ de altura) sugere que o processo de estabelecimento e, principalmente, a colonização são recentes, pois segundo Goldenberg et al. (2005), essas espécies podem atingir 2,0 m de altura na idade adulta. No entanto, as características diferenciadas demonstram que há núcleos de Clidemia urceolata em diferentes estágios de desenvolvimento e que a evolução inicial dos mesmos pode estar ocorrendo de acordo com o crescimento e adensamento dos indivíduos. Esses diferentes estágios de desenvolvimento sugerem um dinamismo dos núcleos de Clidemia urceolata 


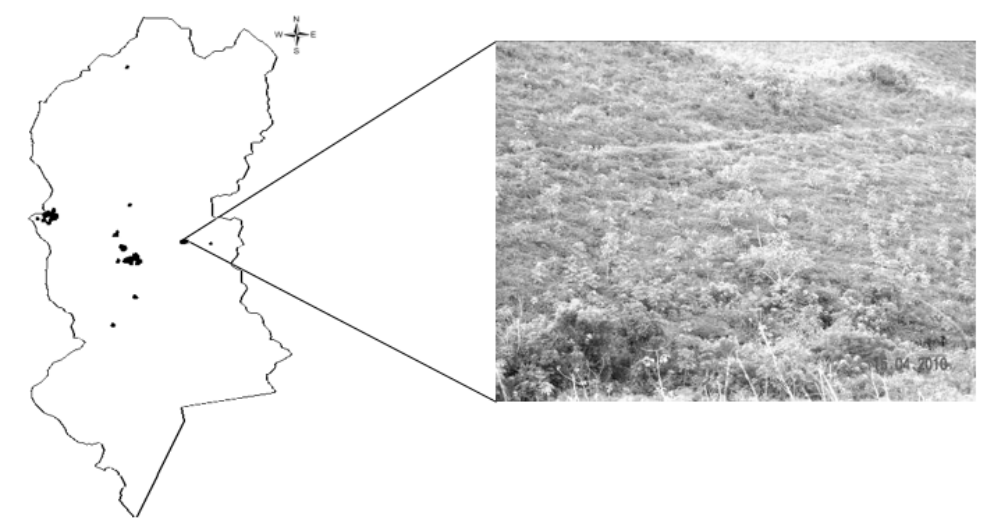

FIGURA 2: Distribuição dos núcleos de Clidemia urceolata DC. georreferenciados na bacia do rio Barra Mansa, Barra Mansa - RJ.

FIGURE 2: Distribution of the nuclei of Clidemia urceolata DC. geo-referenced in the watershed of Barra Mansa River, RJ state.

A

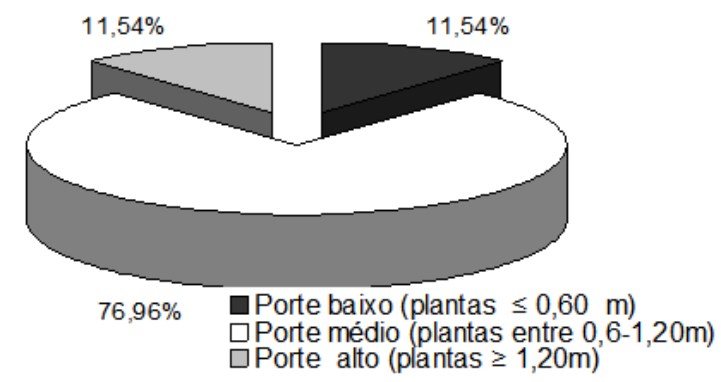

C

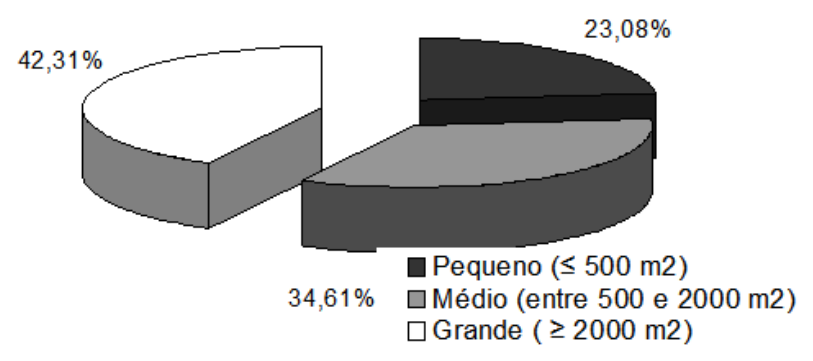

B

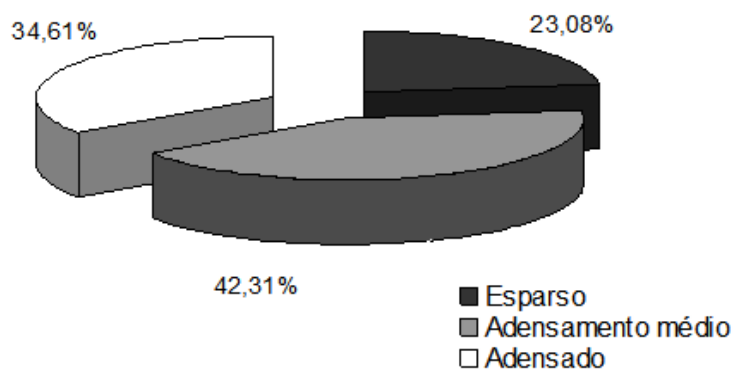

D

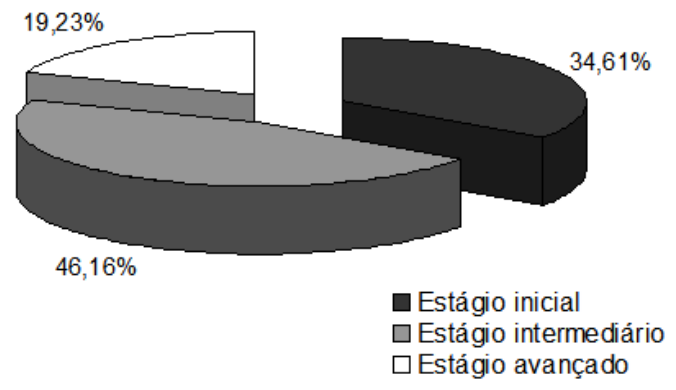

FIGURA 3: Caracterização dos núcleos quanto ao porte das plantas (A), adensamento (B), área (C) e estágio de desenvolvimento (D).

FIGURE 3: Characterization of the nuclei as the size of the plants (A), densification (B), area (C) and development stage (D).

na bacia hidrográfica do rio Barra Mansa, com os núcleos jovens representando a entrada da espécie no sistema (pequenas moitas de Clidemia urceolata em meio à pastagem) e a sua saída quando há o avanço no estágio sucessional.

A capacidade da Clidemia urceolata em colonizar pastagens abandonadas, com solos exauridos após intenso uso (poucos recursos, como água, matéria orgânica e nutrientes; solos compactados e elevada oscilação termica diária) e fogo frequente, de modo a formar pequenas moitas, assim como dominar a fisionomia de capoeiras 
em diferentes estágios de desenvolvimento, possivelmente está associada a estratégias específicas de vida dessa espécie. Estratégias como produção abundante de frutos e sementes durante todo ano, e dispersão zoocórica (observação pessoal) podem contribuir para a dispersão da espécie no tempo e no espaço, assim como para atrair dispersores de propágulos de outras espécies para o seu entorno, o que potencializa o seu papel como nucleadora. Essa dispersão zoocórica é a mais frequente para as espécies da família Melastomataceae (LEVEY, 1990), a qual pertence a Clidemia urceolata. As espécies dessa família são recursos importantes para diferentes populações animais das matas secundárias, principalmente as aves (STILES e ROSSELLI, 1983; POULIN et al. 1999; PEREIRA e MANTOVANI, 2001; ANTONINI e NUNESFREITAS, 2004).

Outra estratégia de colonização de pastagens abandonadas pode estar associada à reprodução vegetativa da Clidemia urceolata, em que grupos de ramos podem estar ligados por raízes subterrâneas. Observações em campo, com escavações de raízes de Clidemia urceolata crescendo em áreas abertas de pastagens abandonadas revelou ramos individuais com sistema radicular isolado e grupos de ramos interligados pelas raízes. Já nas clareiras dos fragmentos foram observados indivíduos de Clidemia urceolata com raízes individuais. Segundo Rosa e Souza (2004), a reprodução vegetativa por meio de raízes é comum em espécies de Cerrado, assim como é constatada em plantas de mata. A ocorrência de reprodução vegetativa já foi observada em espécies de Piper sp., que têm hábito de formar touceiras, principalmente em áreas de borda de mata (ROSA e SOUZA, 2004). Esse tipo de comportamento também foi identificado por Williamson et al. (1998), avaliando as estratégias de colonização da pioneira Vismia sp., em pastagens abandonadas em processo de degradação na Amazônia Central e beiras de estradas na Mata Atlântica, em Linhares ES. Segundo os mesmos autores, o hábito clonal de alguns indivíduos pode ser uma resposta facultativa às perturbações frequentes. Esse mecanismo pode contribuir para o sucesso de colonização dessas espécies em pastagens abandonadas, de forma a resistir à competição de gramíneas, que, segundo Holl et al. (2000), Campos (2010) e Ortega-Pieck et al. (2011), é uma das principais barreiras para o sucesso da regeneração e recuperação desses ambientes.
Ajunção dessas estratégias de colonização contribui para caracterizar a Clidemia urceolata como uma espécie rústica, que, segundo Pott e Pott (2002) e Baylão Junior (2010), é a espécie capaz de colonizar terrenos declivosos, que perderam capacidade de produção agrícola, devido aos seus solos pobres em nutrientes e com baixos teores de matéria orgânica, que, muitas vezes, sofrem frequentes perturbações como fogo, agravando ainda mais a qualidade do solo.

O sucesso de colonização das primeiras pioneiras pode estar associado às características iniciais da área e aos usos anteriores ao abandono das mesmas (WILLIAMSON et al., 1998). Segundo o mesmo autor, uma pioneira bem estabelecida pode reestruturar as funções ecológicas em uma dada região e contribuir para regeneração das espécies florestais sob seu dossel.

Assim como observado para a Clidemia urceolata, outras espécies pioneiras com capacidade nucleadora e potencial para a restauração florestal foram identificadas em outras áreas perturbadas, como em pastagens abandonadas na Costa Rica (SLOCUM e HORVITZ, 2000; HOLL et al. 2000); no município de Guaraqueçaba - PR (CAMPO, 2010) e na região de Piraí - RJ (BAYLÃO JUNIOR, 2010). A capacidade de fornecimento de abrigo e alimento para a fauna dispersora contribui no incremento de sementes em torno dessas plantas (SLOCUM e HORVITZ, 2000; SANTOS e PILLAR, 2007). Segundo Holl et al. (2000); Baylão Junior (2010) e Campos (2010), a elevada regeneração de outras espécies em torno dessas plantas pode contribuir na formação de núcleos de vegetação e no avanço da sucessão, consolidando os processos ecológicos responsáveis pela sustentabilidade da floresta.

A organização em núcleos da vegetação também foi descrita em outros ambientes como na Restinga, como as moitas de Clusias e bromélias (SCARANO, 2002; PIMENTEL et al., 2007). Segundo Scarano (2002), essas espécies fornecem micro-habitats favoráveis, permitindo a germinação e crescimento de outras espécies nesses ambientes inóspitos e com isso possibilitam o avanço sucessional.

Nas pastagens abandonadas, onde os solos apresentam pouca matéria orgânica e horizonte "A" parcialmente erodido, podendo ter restrição para estabelecimento das plantas exigentes, as espécies pioneiras podem ter um papel fundamental na modificação desses ambientes, assim como na facilitação do estabelecimento de 
outras espécies. Nesse foco, Clidemia urceolata pode ser entendida como uma espécie com grande potencial de facilitação da restauração florestal das áreas perturbadas. Para tanto, faz-se necessária uma avaliação mais precisa, utilizando indicadores bióticos e abióticos da sucessão para validar esse potencial.

\section{CONCLUSÕES}

A presença de núcleos de vegetação com dominância fitofisionômica de Clidemia urceolata em 7,9 ha $(0,11 \%)$ dos 6.839 ha da bacia hidrográfica em estudo, assim como a tendência de predomínio de núcleos de porte médio $(76,92 \%)$, pouco adensado (42,31\%), tamanho grande $(42,31 \%)$ e estágio intermediário de desenvolvimento $(46,16 \%)$, evidenciou que os mesmos se encontram em formação, com entrada de mais indivíduos de Clidemia urceolata e outras espécies arbustivas/ arbóreas entrando no sistema da bacia hidrográfica.

A estratégia de vida da Clidemia urceolata caracteriza-a como uma espécie rústica, pois possibilita sua colonização espontânea em áreas de pastagens abandonadas que perderam capacidade de produção agrícola.

A forma de colonização da Clidemia urceolata em pastagens abandonadas (formação de moitas), acrescida de suas estratégias de reprodução e dispersão pode constituir propriedades ecológicas capazes de atrair novas espécies e contribuir para dinamizar a sucessão ecológica, podendo atuar como agente espontâneo de restauração florestal das áreas perturbadas.

\section{AGRADECIMENTOS}

Agradecemos a CAPES pela Bolsa de doutorado no PPGCAF/UFRRJ ao primeiro autor e ao Instituto Federal do Rio de Janeiro, Campus Pinheiral, pelo apoio e infraestrutura.

\section{REFERÊNCIAS BIBLIOGRÁFICAS}

ANTONINI, R. D.; NUNES-FREITAS, A. F. Estrutura populacional e distribuição espacial de Miconia prasina D.C. (Melastomataceae) em duas áreas de Floresta Atlântica na Ilha Grande, RJ, Sudeste do Brasil. Acta Botânica Brasílica, v. 18, n. 3, p. 671-676, 2004.

ARAUJO, M. M. et al. Densidade e composição florística do banco de sementes do solo de florestas
sucessionaisnaregiãodoBaixoRioGuamá,Amazônia Oriental. Scientia Forestalis, Piracicaba. n. 59, p. 115-130, 2001.

BAIDER, C.; TABARELLI, M.; MANTOVANI, W. O banco de sementes de um trecho de floresta Atlântica Montana São Paulo, Brasil. Rev. Brasil. Biol. v. 59, n. 2, p. 319-328, 1999.

BAYLÃO JUNIOR, H.F. Levantamento de espécies com vocação para facilitar processos de restauração espontânea de ecossistemas perturbados na vertente atlântica da Serra do Mar, Piraí - RJ. 2010. 89 f. Dissertação (Mestrado em Ciências Ambientais e Florestais) - Instituto de Floretas, Universidade Federal Rural do Rio de Janeiro, Seropédica, 2010.

BRAGA, A. J. T. et al. Composição do banco de sementes de uma Floresta Semidecidual secundária considerando o seu potencial de uso para recuperação ambiental. Revista Árvore, Voçosa. v. 32, n. 6, p. 1089-1098, 2008.

BUSCHBACHER, R.; UHL, C.; SERRÃO, E. A. S. Abandoned pastures in Eastern Amazônia. II. Nutrient stocks in the soil and vegetation. Journal of Ecology. v. 76, p. 682-699, 1988.

CAMPOS, R. P. Espécies lenhosas pioneiras apresentam diferentes potenciais de facilitação da regeneração natural em pastagens abandonadas? 2010. 39 f. Dissertação (Mestrado em Ecologia) Setor de Ciências Ambientais. Universidade Federal do Paraná, Curitiba, 2010.

CANDIDO, C. P. A família Melastomataceae na Serra do Cabral-MG: Tribos Melastomeae, Merianieae e Miconieae. 2005. 88 f. Dissertação (Mestrado em Biologia Vegetal) - Instituto de Biologia. Universidade Estadual de Campinas, SP, 2005.

CARPANEZZI, A. A. Fundamentos para a reabilitação de ecossistemas florestais. In: GALVÃO, A. P. M.; SILVA, V. P. (Ed.). Restauração florestal: fundamentos e estudos de caso. Colombo: Embrapa Florestas, 2005. p. 27 - 45.

CARVALHO, P. E. R. Técnicas de recuperação e manejo de áreas degradadas. In: Galvão, A. P. M. (Org.). Reflorestamento de propriedades rurais para fins produtivos e ambientais: um guia para ações municipais e regionais. Brasília: Embrapa, 2000. p. 251-268.

CASTRO, C. M.; MELlO, E. V.; PEIXOTO, M. N. O. Tipologia de processos erosivos canalizados e escorregamentos - proposta para avaliação de riscos geomorfológicos urbanos em Barra Mansa (RJ). Anuário do Instituto de Geociências. v. 25, 
p. 11-24, 2002.

CEIVAP- Comitê para Integração da Bacia Hidrográfica do Rio Paraíba do Sul. Diagnóstico da situação atual dos recursos hídricos. In: Plano da bacia do Rio Paraíba do Sul V.1-8. Fundação Coppetec. UFRJ. Agência Nacional das Águas (ANA). 2002, 1200p. Disponível em: http://www. ceivap.org.br/planobacia.htm. Acesso em: 10 junho, 2008.

CHAZDON, R. L. Beyond deforestation: restoring forests and ecosystem services on degraded lands. Science, v. 320, p. 1458-1460, 2008.

CLAUSING, G.; RENNER, S. S. Molecular Phylogenetics of Melastomataceae and Memecylaceae: implications for character evolution. Amerrican Journal of Botany. v. 88, n. 3, p.486-498, 2001.

COPPE-UFRJ. Projeto preparatório para o gerenciamento dos recursos hídricos do Paraíba do Sul: Projeto-piloto de controle de erosão em Barra Mansa (RJ). Rio de Janeiro. 2000. 27 p. Disponível em: <http:// www.ceivap.org.br>. Acesso em: 14 de junho de 2005.

CORTINES, E.; VALCARCEL, R. Influence of pioneer-species combinations on restoration of disturbed ecosystems in the Atlantic Forest, Rio de Janeiro, Brazil. Revista Árvore, v. 33, n. 5, p. 927-936, 2009.

COSTANZA, R. et al. The value of the world's ecosystem services and natural capital. Nature. v. 387, n. 15 , p. 253-260, 1997.

DRUMMOND, J. A. Devastação e preservação ambiental no Rio de Janeiro. Niterói: EDUFF, 1997, $306 \mathrm{p}$.

ENGEL, V. L.; PARROTTA, J. A. Definindo restauração ecológica; tendências e perspectivas mundiais. In: KAGEYAMA, P. Y. et al. (Org.). Restauração ecológica de ecossistemas naturais. Botucatu: FEPAF, 2008. p. 1 - 26.

FIGUEIREDO, A. O. T.; MADRUGA, P. R. A.; PEREIRA, R. S. Geoprocesamiento aplicado al analisis del uso de la tierra em el município de San Piedro de Ycuanadyyú, Paraguai. Ciência Florestal, Santa Maria, mm, v. 12, n. 2, p. 177-185, 2002.

FIGUEIREDO, P. H. A. et al. Avaliação do potencial seminal da Cecropia Pachystachya Trécul no banco de sementes do solo de um fragmento florestal em restauração espontânea na Mata Atlântica, Pinheiral - RJ. Revista Biociências, v. 17, n. 2, p. 43-51, 2011.

FRANCO, B, K. S. Análise da regeneração natural e do banco de sementes em um trecho de floresta estacional semidescidual no campus da Universidade Federal de Viçosa, MG. 2005. 66 f. Dissertação (Mestrado em Ciência Florestal) - Departamento de Engenharia Florestal. Universidade Federal de Viçosa, Viçosa, 2005.

Fundação COPPETEC. Plano de Recursos Hídricos da Bacia do Rio Paraíba do Sul. 2007. 32 p. Disponível em: http://www.cnae.ibge.gov.br/. Acesso em: 02 de dezembro de 2011.

GASPARINO, D. et al. Quantificação do banco de sementes sob diferentes usos do solo em área de domínio ciliar. Revista Árvore, Viçosa, v. 30, n. 1, p. 1-9, 2006.

GOLDENBERG, R.; REGINATO, M. Sinopse da família Melastomataceae na Estação Biológica de Santa Lúcia, Santa Teresa, Espírito Santo. Boletim do Museo de Biologia Mello Leitão. n. 20, p. 33-58, 2006.

GOLDENBERG, R.; SOUZA, C. M. F.; DEQUECH, H. B. Clidemia, Óssea e Pleiochiton (Melastomataceae) no estado do Paraná, Brasil. Hoehnea, v. 32, n. 3, p.453-466, 2005.

HOLL, K. D. et al. Tropical Montane Forest Restoration in Costa Rica: Overcoming Barriers to Dispersal and Establishment. Restoration Ecology, v. 8, n. 4, p. 339-349, 2000.

INMET - Instituto Nacional de Meteorologia. Ministério da Agricultura e Reforma Agrária. Normais Climatológicas (1961-1990). Brasília: SPI/EMBRAPA, 1992. 84p.

INSTITUTO BRASILEIRO DE GEOGRAFIA E ESTATÍSTICA (IBGE). Classificação Nacional de Atividades Econômicas - CNAE. 2.0. Disponível em http://www.cnae.ibge.gov.br/. Acesso em 20 jul. 2011.

LESSA, L. G.; COSTA, F. N. Food habits and seed dispersal by Thrichomys apereoides (Rodentia: Echimyidae) in a brazilian Cerrado Reserve. Mastozoología Neotropical. 2009. Disponivel em: $\quad<\mathrm{http}: / /$ www.sbmz.org/arquivos/socios/ Habitosalimentares/habit-alimentares01.pdf $>$. Acesso em: 10 de dezembro de 2011.

LEVEY, D. J. Habitat-dependent fruiting behaviour of an understory tree, Miconia centrodesma, and tropical treefall gaps as keystone habitats for frugivores in Costa Rica. Journal of Tropical Ecology. v. 6, p. 409-420, 1990.

MARTINS, S. V. et al. Distribuição de espécies arbóreas em um gradiente topográfico de Floresta Estacional Semidescidual em Viçosa, MG. Scientia Forestalis, n. 64, p. 172-181, 2003.

MENEZES, C. E. G. Diagnóstico de degradação 
do solo em função da topografia e cobertura vegetal no município de Pinheiral. 1999. $186 \mathrm{f}$. Dissertação (Mestrado em Agronomia) - Instituto de Agronomia, Universidade Federal Rural do Rio de Janeiro, Seropédica, 1999.

MENEZES, C. E. G. Integridade de paisagem, manejo e atributos do solo no Médio Vale do Paraiba do Sul, Pinheiral-RJ. 2008. 164 f. Tese (Doutorado em Agronomia) - Universidade Federal Rural do Rio de Janeiro, Seropédica, 2008.

MICHELANGELI, F. A., REGINATO, M. Clidemia. In: Lista de espécies da Flora do Brasil. Jardim Botânico do Rio de Janeiro. 2010. Disponível em: http://floradobrasil.jbrj.gov.br/2010/FB009452. Acesso: em julho de 2011.

MIRANDA, C. C. et al. Avaliação das preferências ecológicas Clidemia urceolata DC. em ecossistemas perturbados. Revista Árvore. v. 35, n. 5, p. 1135-1144, 2011.

MIRITI, M. N. Regeneração florestal em pastagens abandonadas na Amazônia central: competição, predação e dispersão de sementes. In: GASCON, C.; MOUTINHO, P (Ed.). Florestas Amazônicas: Dinâmica, Regeneração e Manejo. Manaus: Instituto Nacional de pesquisas da Amazônia, 1998. p.179-190.

MOUTINHO, P. R. S. Impactos da formação de pastagens sobre fauna de formiga: consequências para a recuperação florestal na Amazônia oriental. In: GASCON, C.; MOUTINHO, P (Ed.). Florestas Amazônicas: Dinâmica, Regeneração e Manejo. Manaus: Instituto Nacional de pesquisas da Amazônia, 1998. p.155-170.

ORTEGA-PIECK, A. et al. Early seedling establishment of two tropical montane cloud forest tree species: The role of native and exotic grasses. Forest Ecology and Management. v. 261, n. 7, p. 1336-1343, 2011.

PEREIRA, T. S.; MANTOVANI, W. Maturação e dispersão de Miconia cinnamomifolia (DC) Naud. na Reserva Biológica de Poço das Antas, município de Silva Jardim, RJ, Brasil. Acta Botânica Brasílica. v. 15 , n. 3, p. 335-348, 2001.

PIMENTEL, M. C. P. et al. Spatial variation in the structure and floristic composition of "restinga" vegetation in southeastern Brazil. Revista Brasileira de Botânica, v. 30, n. 3, p. 543-551, 2007.

POTT, A.; POTT, V. J. Plantas nativas para recuperação de áreas degradadas e reposição da vegetação no Mato Grosso do Sul. EMBRAPA gado de corte. Campo Grande - MS. 2002. (Comunicado Técnico - EMBRAPA. n. 75)
POULIN, B. et al. Interspecific synchrony and asynchrony in the fruiting phenologies of congeneric bird-dispersed plants in Panama. Journal of Tropical Ecology. v. 15, p. 213-227, 1999.

RADAMBRASIL. Levantamento dos recursos Naturais: folha SF. 23/24 Rio de Janeiro/Vitória: Ministério de Minas e Energia, Rio de Janeiro: 1983. v.32. $780 \mathrm{p}$.

RENNER, S. S. Phylogeny and classification of the Melastomataceae and Memecylaceae. Nordic Journal of Botany. v. 13, n. 5, p. 519-540, 1993.

ROPPA, C. Avaliação da dinâmica da restauração de ecossistemas perturbados da mata atlântica em uma região de exíguos atributos ambientais, Nova Iguaçu - RJ. 2009. 140 f. Dissertação (Mestrado em Ciências Ambientais e Florestais) - Universidade Federal Rural do Rio de Janeiro, Seropédica, 2009.

ROSA, S. M.; SOUZA, L. A. Estruturas de reprodução de Piper amalago VAR. medium Linnaeus (Piperaceae). Acta Científica Venezolana. v. 55, p. 27-34. 2004.

SANTOS, M. M. G.; PILLAR, V. D. Influência de Poleiros Naturais e Artificiais na Expansão da Floresta com Araucária sobre os Campos, em São Francisco de Paula, RS. Revista Brasileira de Biociências, v. 5, n. 1, p. 594-596, 2007.

SCARANO, F. R. Structure and floristic relationships of plant communities in stressful habitats marginal to the Brazilian Atlantic Rainforest. Annals of Botanic, v. 90, p. 517-524, 2002.

SLOCUM, M. G.; HORVITZ, C. C. Seed arrival under different genera of trees in a neotropical pasture. Plant Ecology, v.149, p. 51-62, 2000.

STILES, F. G.; ROSSELLI, L. Consumption of fruits of the Melastomataceae by birds: how diffuse is coevolution? Vegetatio. v. 107/108, p. 57-75, 1983.

TOLEDO, L. O.; PEREIRA, M. G. Dinâmica da deposição de serrapilheira em florestas secundárias do município de Pinheiral, RJ. Floresta e Ambiente. v. 11, n. 1. p. 39-46, 2004.

TRÊS, D. R. et al. Banco e Chuva de Sementes como Indicadores para a Restauração Ecológica de Matas Ciliares. Revista Brasileira de Biociências. v. 5, n. 1, p. 309-311, 2007.

UHL, C. ; BUSCHBACHER, R.; SERRÃO, E. A. S. Abandoned pastures in eastern Amazonia. I. Patterns of plant succession. Jounal of Ecology. v.76, p. 663-681. 1988.

VALCACEL, R. Proposta de ação para o manejo de 
bacia hidrográfica do Rio Paraíba do Sul. Floresta e Ambiente. v. 2. n. 2, p. 129-131, 1995.

VALCARCEL, R.; SILVA, Z. S. A eficiência conservacionista de medidas de recuperação de áreas degradadas: proposta metodológica. Floresta. v. 21, n. $1 / 2$, p. $101-114,2000$.

WILLIAMSON, G. B. et al. Estratégias de colonização de árvores pioneiras nos Neotrópicos. In: GASCON, C.; MOUTINHO, P (Ed.). Florestas
Amazônicas: Dinâmica, Regeneração e Manejo. Manaus: Instituto Nacional de pesquisas da Amazônia, 1998. p.131-144.

WURDACK, J. J., MORLEY, T.; RENNER, S. Melastomataceae. In: A. R. A. Görts van Rijn (ed.). Flora of the Guianas. Germany: Koeltz Scientific Books, 1993, v. 99, 425 p.

ZAR, J. H. Biostatistical analysis. 4th ed. New Jersey: Prentice-Hall, Inc. 1999. 663 p. 